\title{
THE RADIOLOGICAL CHANGES IN PULMONARY VENOUS HYPERTENSION, WITH SPECIAL REFERENCE TO THE ROOT SHADOWS AND LOBULAR PATTERN
}

\author{
BY
}

\author{
H. R. S. HARLEY \\ From Sully Hospital Thoracic Centre, Cardiff
}

Received May 14, 1960

\begin{abstract}
Despite all that has been written about the radiological changes produced by mitral or pulmonary venous obstruction, the differences caused by a raised pressure in the pulmonary veins on the one hand and in the pulmonary arteries on the other are not always appreciated. The purpose of this communication is to clarify these differences. The observations are based largely on a study of the radiograms taken before and after valvotomy performed on 173 patients suffering from mitral stenosis, in all of whom pressure records were made at the time of operation, and some of whom were also catheterized. A full account of the hæmodynamic consequences of pulmonary venous hypertension has been given elsewhere (Harley, 1960).

The unqualified term pulmonary hypertension should be discarded in favour of the more specific ones pulmonary venous hypertension, pulmonary mixed hypertension, and pulmonary arterial hypertension. In the following discussion the first of these terms is used to describe the condition in which the pulmonary venous pressure is raised, but the pulmonary vascular resistance is normal or only slightly increased, and any pulmonary arterial hypertension is purely passive. By pulmonary mixed hypertension is meant pulmonary venous hypertension complicated by a rise of pulmonary vascular resistance due to constriction of the small and medium sized pulmonary arteries and arterioles, spastic or structural, causing active or obstructive pulmonary arterial hypertension. In pulmonary venous hypertension the pulmonary pressure gradient is within normal limits whereas in mixed hypertension it is raised. By pulmonary arterial hypertension is meant a raised pulmonary arterial pressure due to causes other than pulmonary venous hypertension. Each of these conditions gives rise to different radiological appearances, which can be distinguished from each other, and also from those caused by pulmonary pleonæmia.
\end{abstract}

\section{Pulmonary Venous Hypertension}

The radiological changes of pulmonary venous hypertension may be described under four headings, namely: pulmonary œdema, structural changes in the interlobular septa, changes in the small and medium sized pulmonary arteries and veins, and changes in the root shadows. Changes in the heart, aorta, and main pulmonary artery will not receive special consideration.

Pulmonary Interstitial Edema. Pulmonary venous hypertension results in a raised capillary pressure which may cause alveolar or interstitial odema of the lung (Parker and Weiss, 1936; Grainger and Hearn, 1935; Doyle et al., 1957; Harley, 1960), but only the interstitial variety will be discussed here. The radiological changes by which this is recognized are loss of translucency, peripheral horizontal lines, and a lobular pattern in the lower parts of the lung fields. Loss of translucency was described by Davies et al. (1953) and by Goodwin (1958). Horizontal lines have 
been discussed by many workers (Kerley, 1933 and 1951; Fleischner and Reiner, 1954; Whitaker and Lodge, 1954; Carmichael et al., 1954; Short, 1955; Grainger and Hearn, 1955; Gough, 1955; Rossall and Gunning, 1956; Grainger, 1958; and Fleming and Simon, 1958), but the only reference to the appearance which I have named the lobular pattern is made by Gough (1957-58) who described a mosaic.

The loss of translucency, the septal lines, and the lobular pattern are seen best in the lower and dependent areas where the hydrostatic pressure is greatest (Fig. 1, 2). Dock (1947) demonstrated that the venous pressure is $20 \mathrm{~cm}$. of water higher at the base than at the apex of the chest when the subject is upright. The "B" lines of Kerley (1951), for which Short (1955) suggested the excellent name of septal lines, and the lobular pattern are due to opacification of the interlobular septa of the lungs. That this is so is made obvious by a study of Gough's thin paper-mounted whole lung sections (Gough, 1957-58), as shown in Fig. 3, which indicate that the septa correspond in length, number, and position with the B lines and with the lobular pattern seen on the radiograms.

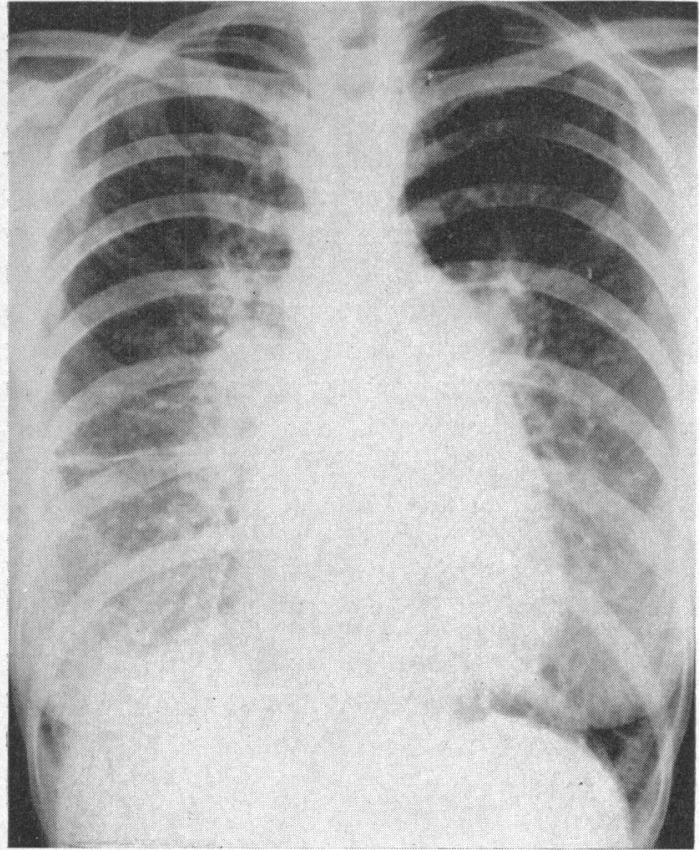

FIG. 1(a).- Mitral stenosis with moderately high pulmonary vascular resistance (PVR). At thoracotomy left atrial pressure (LAP) $24 \mathrm{~mm}$. $\mathrm{Hg}$ and pulmonary artery pressure (PAP) $32 / 20 \mathrm{~mm}$. $\mathrm{Hg}$. Florid pulmonary venous hypertension with venous root shadows, loss of translucency, wide horizontal lines, and well marked lobular pattern.

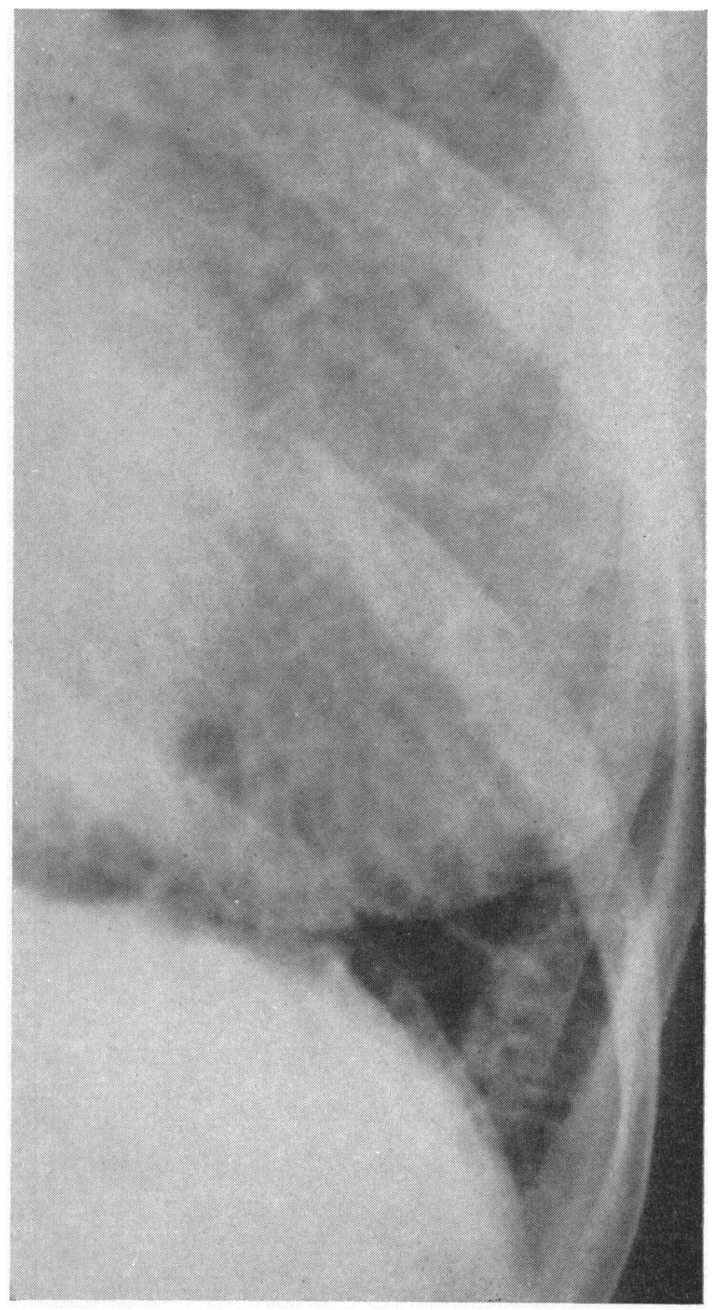

Fig. 1(b)-Enlargement of left lower zone to show lobular pattern. 


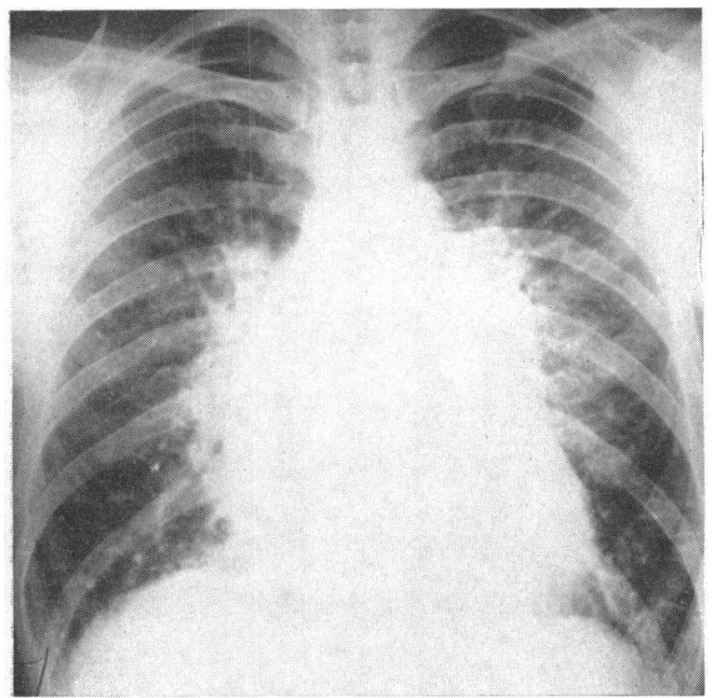

FIG. 2(a).-Pure mitral stenosis with slight aortic stenosis and high PVR. At thoracotomy LAP $18 \mathrm{~mm} . \mathrm{Hg}$ and PAP $68 / 48 \mathrm{~mm}$. $\mathrm{Hg}$. At catheterization PAP $100 / 45 \mathrm{~mm}$. Hg. Root shadows show mixed arterial and venous pattern, the left being more arterial than the right. Lower zone constriction contrasts with upper zone dilatation. Well marked thick and thin horizontal lines, but no lobular pattern and little loss of translucency.

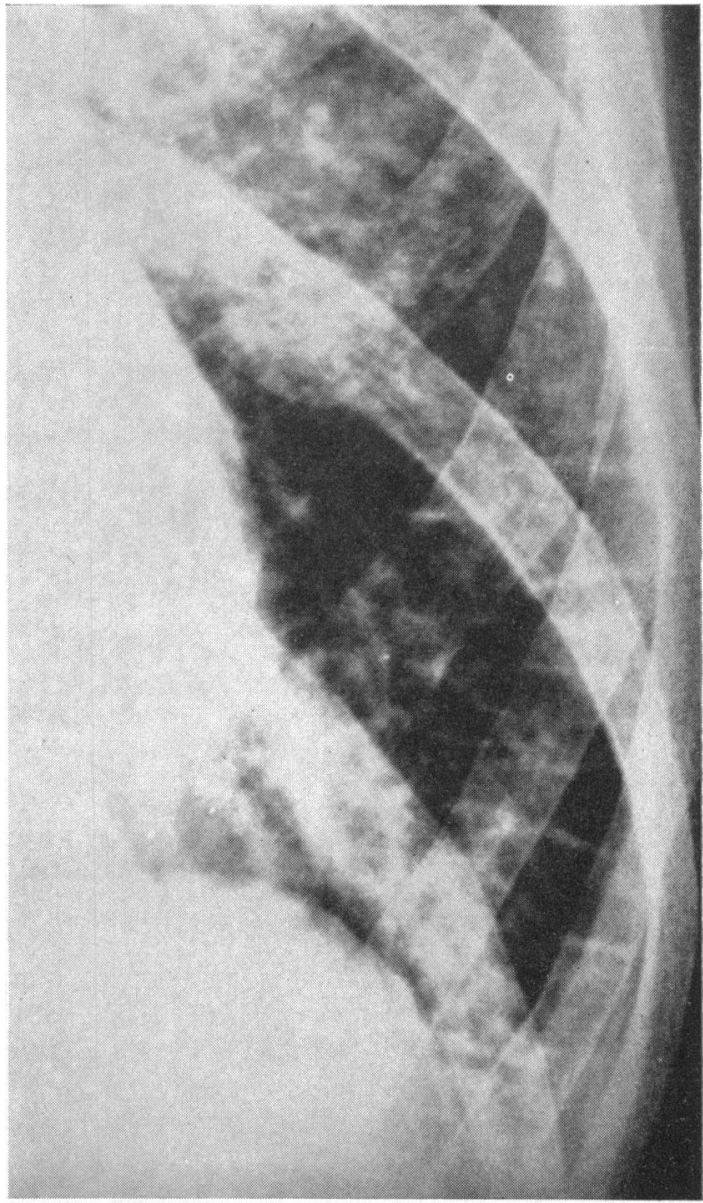

Fig. 2(b).-Enlargement to show horizontal lines in left lower zone.

The evidence is good that loss of translucency and opacification of the interlobular septa are due to interstitial œedema. These changes occur in the dependent areas where capillary pressure is highest in the upright position (Dock, 1947). The changes are extremely variable from time to time, becoming greater with activity and less with rest (Harley, 1960). There is a striking difference in the radiograms taken when patients attend at outpatients before valvotomy compared with those taken just before the operation after they have been in hospital for a period. This is well shown in Fig. 5. Striking improvement results from valvotomy also. These changes have been seen in a variety of conditions that cause a rise in pulmonary capillary pressure. Edema of the interlobular septa has been demonstrated microscopically by Parker and Weiss (1936), by Gough (1955), and also by my colleagues Parry and Storring (personal communication) in biopsy specimens removed from the lung by the author at the time of valvotomy for mitral stenosis (Fig. 4). Finally a relationship can be demonstrated between these changes and the left atrial pressure, measured directly or indirectly (Grainger and Hearn, 1955; Rossall and Gunning, 1956). In 173 cases of mitral stenosis in which the writer measured the left atrial pressure by needle puncture at thoracotomy, horizontal lines were widely scattered over all ranges of pressure, but were more common in the higher pressure groups (Harley, 1960). When the mean left atrial pressure was $21 \mathrm{~mm}$. $\mathbf{H g}$ 


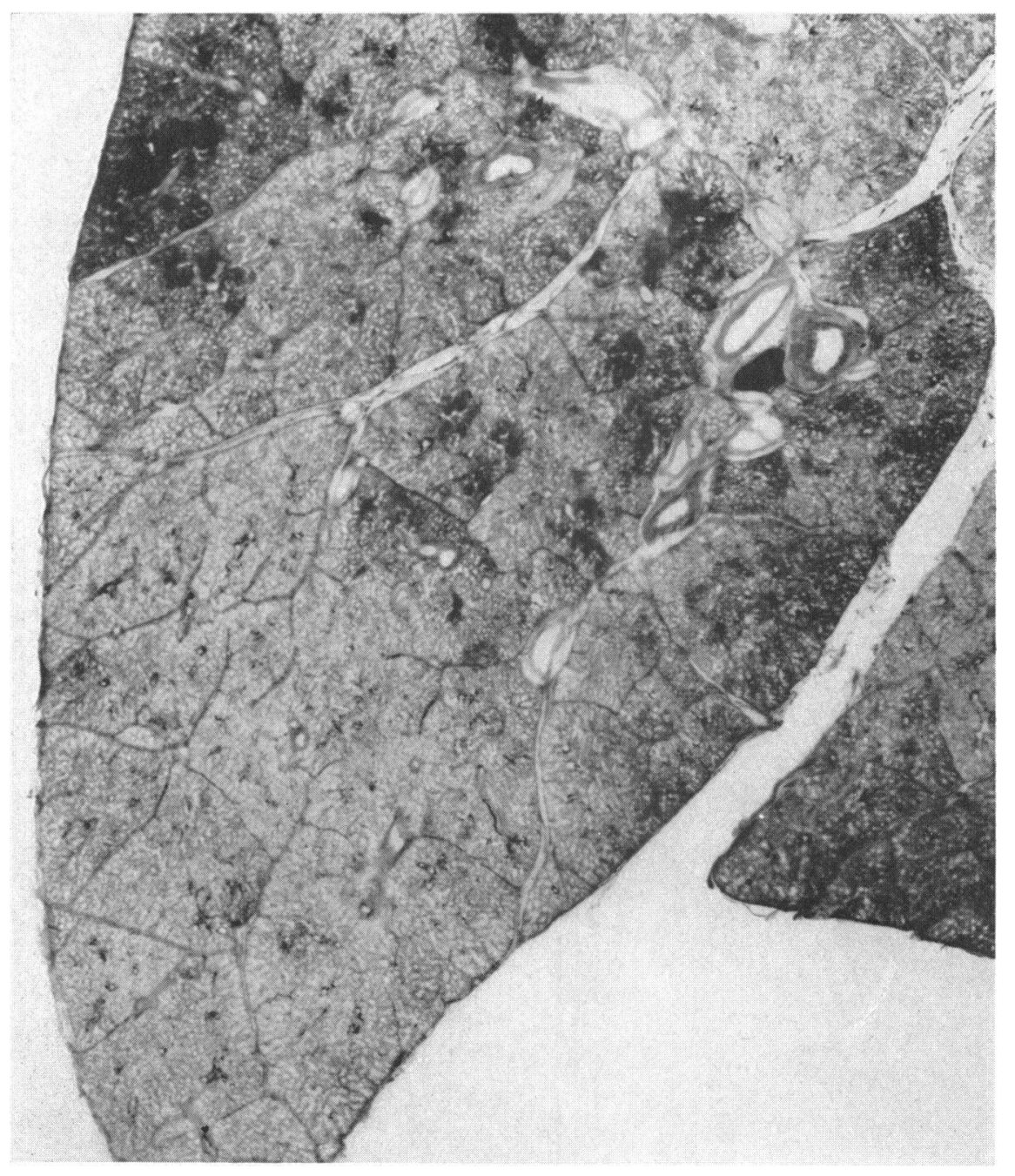

FIG. 3.-Thin paper-mounted section of lower lobe of lung, showing interlobular septa outlined by hæmosiderin. Their relationship to radiological horizontal lines and lobular pattern is obvious. (By the courtesy of Professor J. Gough.)

or more the percentage of cases showing septal lines was 42 , whereas when the pressure was less the percentage dropped to 24 . The wide scatter is due to two facts; firstly, as already indicated, the left atrial and pulmonary venous pressure fluctuates widely from time to time, and secondly all horizontal lines are not due to interstitial œdema (vide infra). Catheterization examinations indicate that septal lines are usually associated with mean left atrial pressures of 17 to $18 \mathrm{~mm} . \mathrm{Hg}$ or more.

Although loss of translucency and horizontal lines are well known signs of pulmonary interstitial œdema, the lobular pattern has received scant attention. This appearance is easily seen in the lower zone in some cases of mitral stenosis and it outlines the secondary anatomical lobules of Miller (1947). It was found by the writer in $45(26 \%)$ of 173 patients with mitral stenosis, and it invariably disappeared after valvotomy. It is illustrated in Fig. 1. The B lines of Kerley (1951) and the lobular pattern represent those interlobular septa that lie in a plane more or less parallel with that of the roentgen rays so that they are rendered opaque (Fig. 1, 2, and 3). 


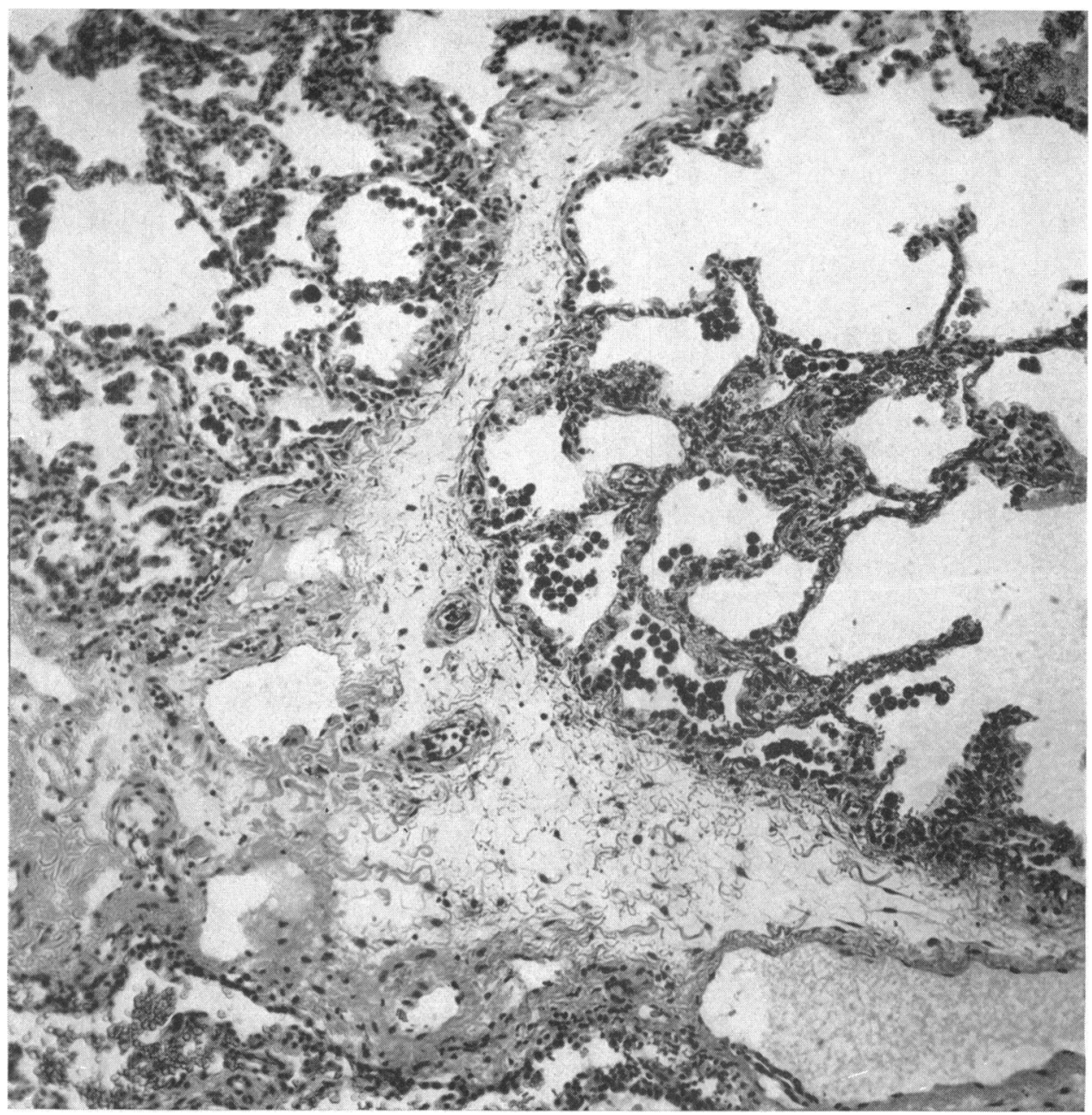

FIG. 4.- Section of lung, showing cedema of interlobular septa, dilated lymphatic vessels, and thickened alveolar septa. Radiograms showed wide horizontal lines and lobular pattern which disappeared within three weeks of valvotomy. Woman of 34 with mitral stenosis, moderate mitral regurgitation, and high pulmonary vascular resistance. At thoracotomy the mean left atrial pressure was $30 \mathrm{~mm}$. $\mathrm{Hg}(\mathrm{H} \& \mathrm{E}: \times 140)$. (By courtesy of Dr. T. E. Parry and F. K. Storring.)

Loss of translucency was evident in the radiograms of 101 out of 173 patients $(59 \%)$ submitted to valvotomy, but is not always an easy sign to interpret because of the shadows thrown by the breasts. That this is, however, a common feature can be seen by comparing radiograms taken by a similar technique before and after successful valvotomy.

Loss of translucency nearly always disappeared after successful valvotomy and the lobular pattern almost invariably did so, but peripheral horizontal lines, which are more or less perpendicular to the pleural surface, did not always do so. It was concluded that loss of translucency and the lobular pattern were caused by interstitial œdema, but that horizontal lines were not always due to this cause. Horizontal septal lines were present in 66 of the 173 patients $(38 \%)$ : they are more commonly seen than the lobular pattern because the subpleural interlobular septa of the lower parts of the lungs are placed nearly in the horizontal plane and are, therefore, close to the plane of travel of the roentgen rays. 


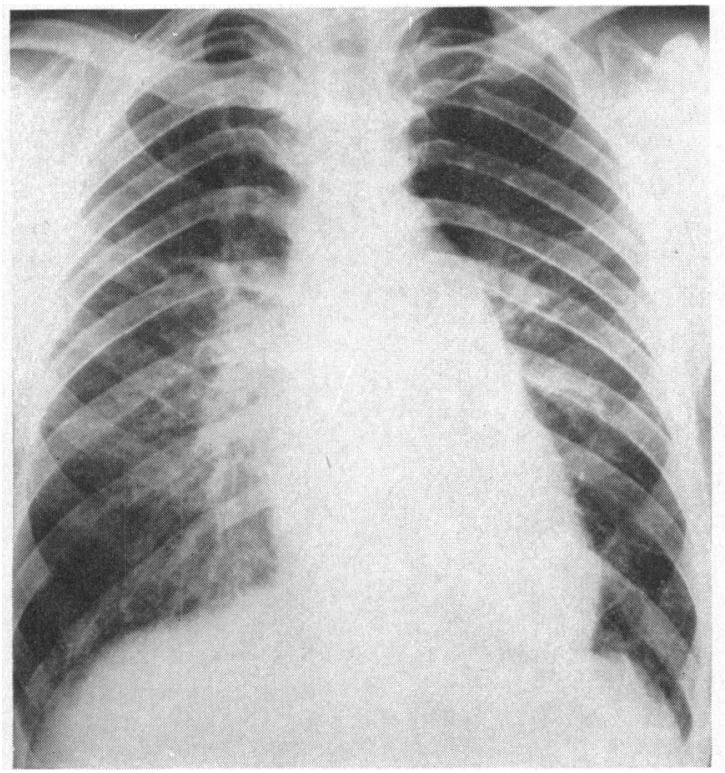

FIG. 5(a).-Pure mitral stenosis, with minimal aortic stenosis and regurgitation, and low PVR. At thoracotomy, LAP $6 \mathrm{~mm}$. Hg and PAP $34 / 18 \mathrm{~mm}$. Hg. Two and one half months before valvotomy typical venous root shadows and fine horizontal lines are seen, but no loss of translucency or lobular pattern. Vessels to lower zones are not constricted and there is much opacity to right of right atrium.

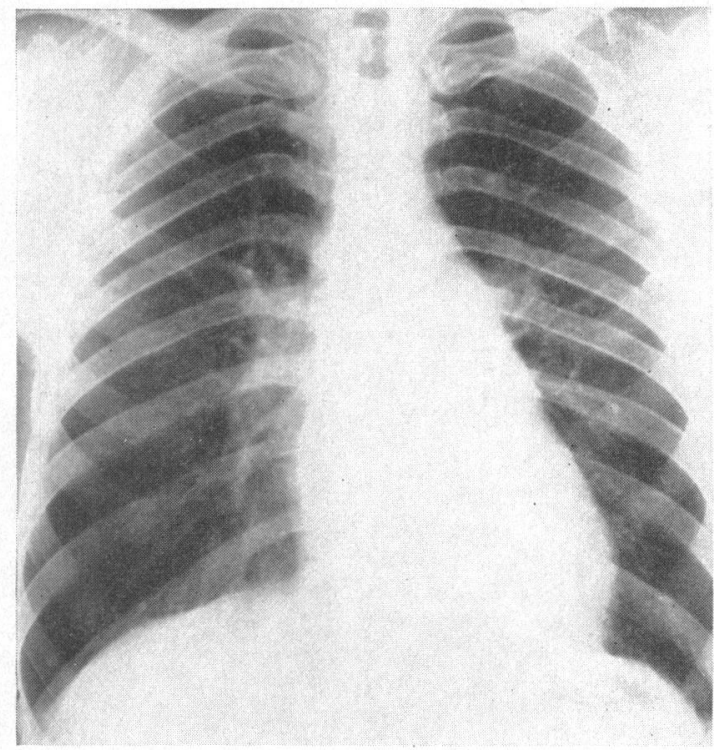

FIG. 5(b).-Remarkable improvement of changes in root shadows and lung fields about two months later, 12 days before valvotomy.

\section{Structural Changes in the Interlobular Septa}

Not all horizontal peripheral septal lines in pulmonary venous hypertension are due to interstitial œdema, for some of them persist after mitral valvotomy. The writer classifies septal lines into reversible or transient and irreversible or permanent. Reversible lines are caused by interstitial œdema (Fig. 4) and disappear after relief of the raised capillary pressure, but irreversible ones are caused by structural changes in the interlobular septa and do not disappear (Fig. 6).

Gough (personal communication) suggested that thick lines might be due to odema and thin ones to the deposition of hæmosiderin in the interlobular septa. To test this hypothesis the writer divided septal lines into thin ones with a thickness of $1 \mathrm{~mm}$. or less, and thick ones wider than this. It was found that both thick and thin lines might persist or disappear after valvotomy, though thick ones were more likely to be reversible. Thick lines were present in 40 of the 173 patients and they disappeared after valvotomy in 24 of the $32(75 \%)$ in whom information was available. Thin lines were present in 53 cases and they disappeared in 24 of the $42(57 \%)$ in whom information was obtained. This investigation shows that the thickness of septal lines is no index of their pathology. Both thick and thin lines may be present in the same patient.

Both Gough (1955) and Fleischner and Reiner (1954) demonstrated hæmosiderin deposition along the borders of the interlobular septa in cases of mitral stenosis, and the latter workers also showed that fibrous thickening was frequent in chronically odematous septa. Fibrous thickening of the septa has been confirmed by Parry and Storring in biopsy specimens removed from the lungs and by the writer at the time of mitral valvotomy (Fig. 6). It seems reasonable to conclude that persistent fine lines are caused by hæmosiderin deposition, and that they are analogous to the septal lines due to pigment deposition seen in cases of tin smelter's and coal miner's pneumoconiosis, 


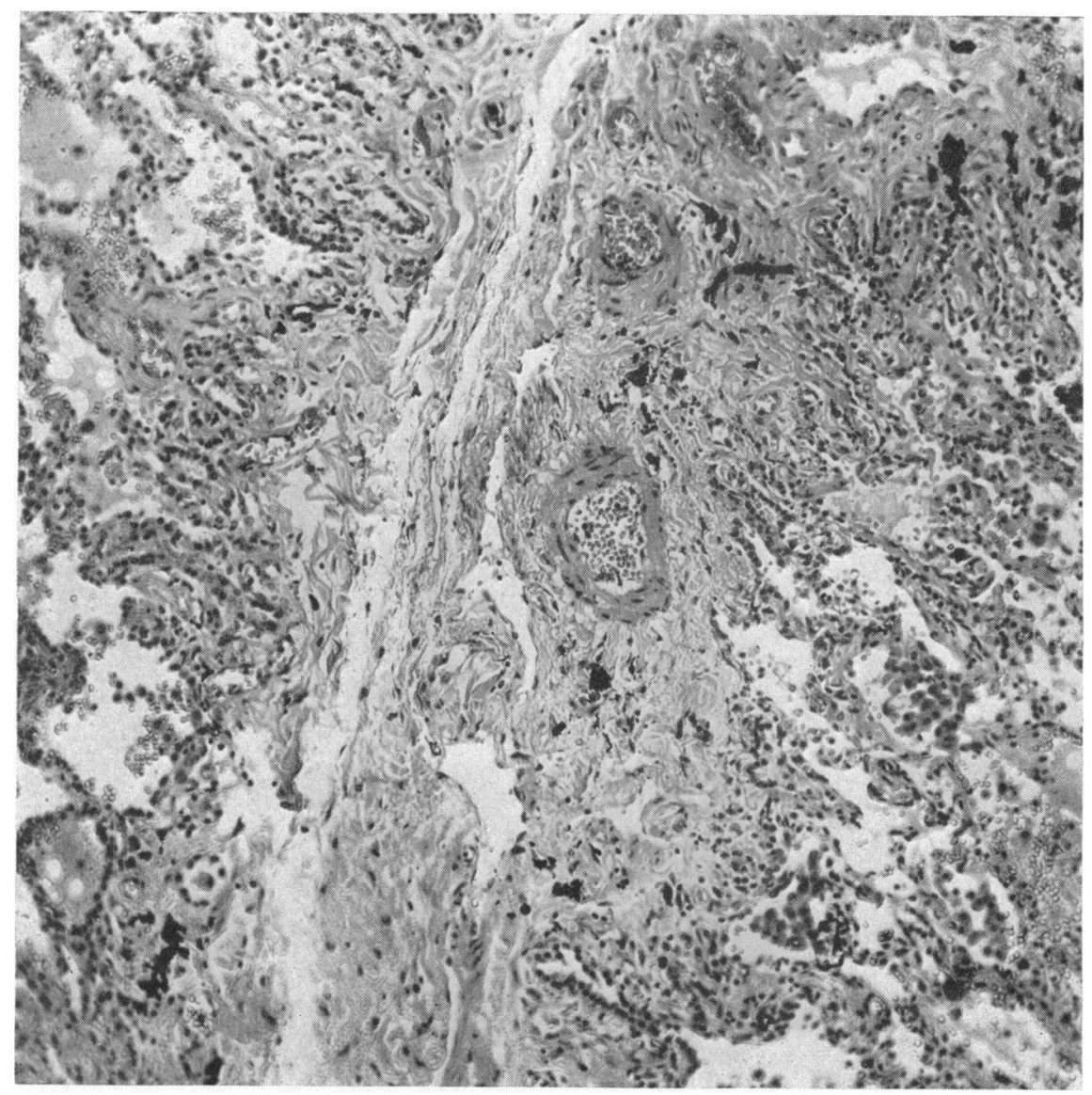

Fig. 6.-Section of lung, showing fibrosed interlobular septum. Case illustrated in Fig. 2. The thick and thin horizontal lines present before valvotomy persisted after operation. (H. \& E.: $\times 140$.) (By courtesy of Dr. T. E. Parry and F. K. Storring.)

while persistent wide lines are the result of fibrous thickening of the interlobular septa. Both of these pathological changes are, of course, end results of chronic pulmonary venous and capillary hypertension, but they afford an adequate explanation of the persistence of septal lines after relief of mitral obstruction. These pathological changes might produce an irreversible lobular pattern but the writer has seen such a pattern persist after successful valvotomy on only one occasion.

\section{Changes in the Small and Medium-sized Pulmonary Arteries and Veins}

One of the consequences of pulmonary venous hypertension is a veno-arterial constriction of the medium-sized and small pulmonary veins and arteries in the lower zone, and this is accompanied by engorgement of the corresponding vessels in the upper zone. The contrast between the dilated vessels in the upper zone and the narrow ones in the lower zone is a striking feature of the radiograms of many cases of mitral stenosis (Fig. 7), and is a valuable radiological sign of pulmonary venous or pulmonary mixed hypertension, for it does not occur in pulmonary arterial hypertension or in pulmonary pleonæmia.

The radiological and arteriographic studies of Goodwin et al. (1952), Davies et al. (1953), Steiner and Goodwin (1954), Doyle et al. (1957), Steiner (1958), Simon (1958), and others have 


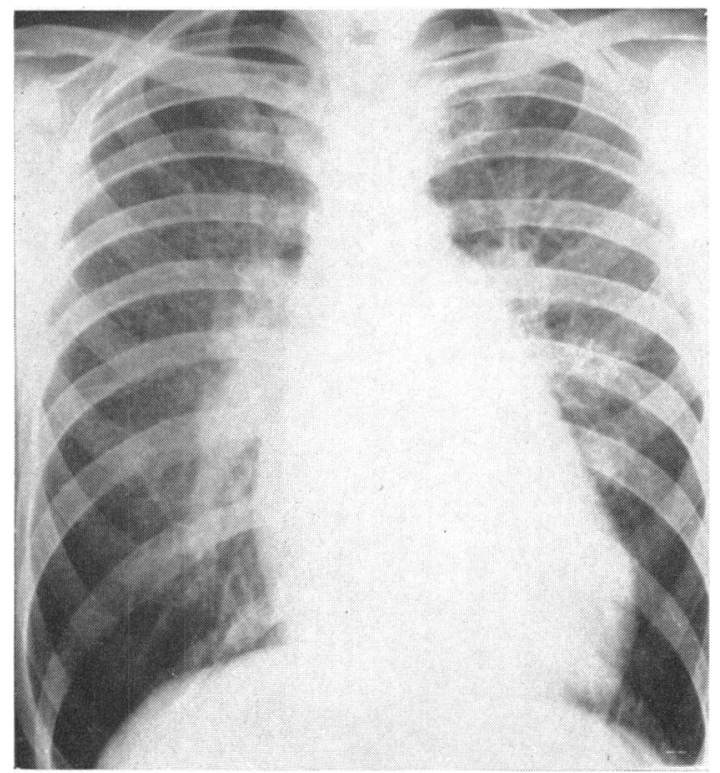

FIG. 7.-Mitral stenosis with considerable regurgitation and aortic stenosis and regurgitation. High PVR (14.2 units). At thoracotomy, LAP $32 \mathrm{~mm}$. $\mathrm{Hg}$ and PAP $55 / 57 \mathrm{~mm}$. $\mathrm{Hg}$. At catheterization, PAP 140/60 mm. Hg. Typical arterial root shadows, venoarterial constriction in lower zone, and slight dilatation of vessels in upper zone.

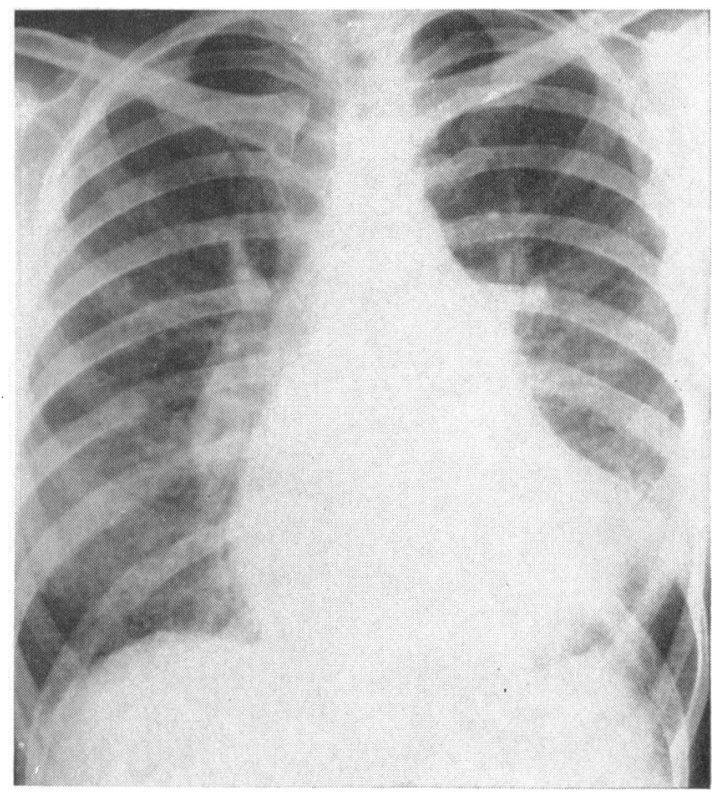

Fig. 8.-Pure mitral stenosis, high PVR, with pulmonary regurgitation, and some aortic stenosis. At thoracotomy, LAP $12 \mathrm{~mm}$. $\mathrm{Hg}$ and PAP 70/38 mm. $\mathrm{Hg}$. At catheterization, PAP $120 / 60 \mathrm{~mm}$. Hg. Typical arterial lung roots, lower zone venoarterial constriction, and thick and thin horizontal lines.

shown that in mitral stenosis the segmental and smaller arteries and arterioles to the lower zones of the lungs are normal in calibre or constricted, whereas those to the upper zones are normal in size or dilated. This has been confirmed by the author. Microscopic and arteriographic studies on post-mortem material by Short (1956) and Harrison (1958) indicate that constriction of both arteries and veins affects particularly the basal segments of the lower lobes, the middle lobe, and the lingula, all of which are below the left atrium, in the erect posture. Short also demonstrated that structural narrowing could occur without evidence of histological change in the vessel walls.

Doyle et al. (1947) suggested that the zonal constriction was activated by pulmonary venous hypertension, the lower zone being affected first because of the hydrostatic increment of pressure. For this reason the change is zonal rather than segmental. According to Doyle and his colleagues venous constriction occurs first and is followed by arterial constriction.

Whitaker and Lodge (1954) and Short (1955) found no relation between the degree of pulmonary venous engorgement of the upper zone and the pulmonary venous pressure, whereas Simon (1958) concluded that as the pulmonary venous pressure rose to 24 to $26 \mathrm{~mm}$. Hg the engorgement in the upper zone increased to a maximum, but that thereafter it decreased with further rises of pressure. Simon concluded that lower zonal constriction redistributed blood to the upper zone where it produced venous engorgement. A further rise in the venous pressure caused constriction to spread to the upper zone, so that engorgement was reduced. If this theory is correct lower zone constriction should precede upper zone dilatation. Wade et al. (1956) thought that the veno-arterial constriction depended upon a local reflex not under autonomic control, but Davies et al. (1953), Balchum et al. (1957), and Goodwin et al. (1958) brought forward evidence to involve an autonomic nervous reflex. 
The writer found dilatation of segmental, subsegmental, and smaller vessels in the upper zones in the radiograms of 98 of 173 patients with mitral stenosis $(57 \%)$. The left atrial pressure measured at thoracotomy in these cases was found to bear no relation to the presence or degree of vascular dilatation in the upper zones, for this occurred in all the pressure ranges. By contrast, constriction in the lower zone occurred in 49 cases $(28 \%)$ and its incidence increased with the height of the left atrial pressure until the latter reached $26 \mathrm{~mm}$. $\mathrm{Hg}$. Thereafter the incidence of constriction remained fairly constant about 50 per cent (Harley, 1960).

A relationship was also found with the pulmonary vascular resistance, the pulmonary pressure gradient, and the pulmonary arterial pressure (Harley, 1960). Thus when lower zonal constriction was present, the pulmonary pressure gradient averaged $18 \mathrm{~mm}$. $\mathrm{Hg}$, but when it was absent the gradient averaged only $11 \mathrm{~mm} . \mathrm{Hg}$. In these two groups the average pulmonary arterial pressures were 51/31 (41 mean) and 38/21 (28 mean) respectively. Furthermore right ventricular hypertrophy was demonstrated by the electrocardiogram in 59 per cent of cases with contriction against 35 per cent of those without it.

The writer's investigations indicate that upper zonal dilatation of the medium-sized and small arteries and veins is common, that it occurs early, and that it is independent of the degree of elevation of the left atrial pressure. It does not appear to be secondary to lower zonal constriction, which is a less common and later change and increases in frequency as the left atrial pressure rises to about $26 \mathrm{~mm}$. Hg. This constriction is associated with, and is presumably at least partially responsible for, a rise in pulmonary vascular resistance, and is seen best in cases of mixed pulmonary venous and arterial hypertension.

\section{Changes in the Root Shadows}

The root shadows undergo important changes in pulmonary venous hypertension, the character of which alters significantly when the hypertension becomes mixed. The appearance of the root shadows gives useful information in distinguishing between the venous, mixed, and arterial varieties of pulmonary hypertension (Fig. 1, 2, 7, 8, and 9).

In pure pulmonary venous hypertension the root shadows become larger and denser than normal, their upper and outer margins become ill-defined and irregular, and their lower ends become broad and prolonged downwards into the very obvious vascular markings of the paracardial regions (Fig. 1a and 5a). On the right side the characteristic space, normally seen between the inner margin of the root shadow and the right atrium, is obliterated. These appearances are chiefly due to enlargement of the left atrium and of the superior and inferior pulmonary veins and their branches down to the intersegmental level. The enlargement of the left atrium and of the right superior and inferior pulmonary veins obliterates the clear space between the inner margin of the right lung root and the right border of the heart. The enlarged proximal branches of the superior pulmonary veins cross the lines of the right and left pulmonary arteries and their descending branches, and fan out into the lung, running forwards, laterally, and backwards, to form significant opacities in the region of the lung roots. The radiation of these enlarged veins in many planes of space obliterates the sharp contours of the upper and outer borders of the right and left root shadows and causes them to lose their definition. In the normal subject these borders are formed by the right and left pulmonary arteries and their lower lobe branches, and are well-defined because these arteries lie almost in one plane, and the veins that cross them throw insignificant shadows. This appearance is not due to perihilar œdema, and œdema is not found if the hilar vessels are dissected at thoracotomy. The wide prolongation of the lower ends of the two root shadows are caused by the opacities produced by the proximal parts of the arteries and veins to the lower and middle lobes and the lingula. The irregularity and loss of definition of the upper and outer border of the root shadows may be accentuated by enlargement of the lobar and segmental arteries to the upper lobes.

When obstructive arterial hypertension complicates pulmonary venous hypertension to cause pulmonary mixed hypertension the appearance of the lung roots changes significantly (Fig. 7 and 8). 


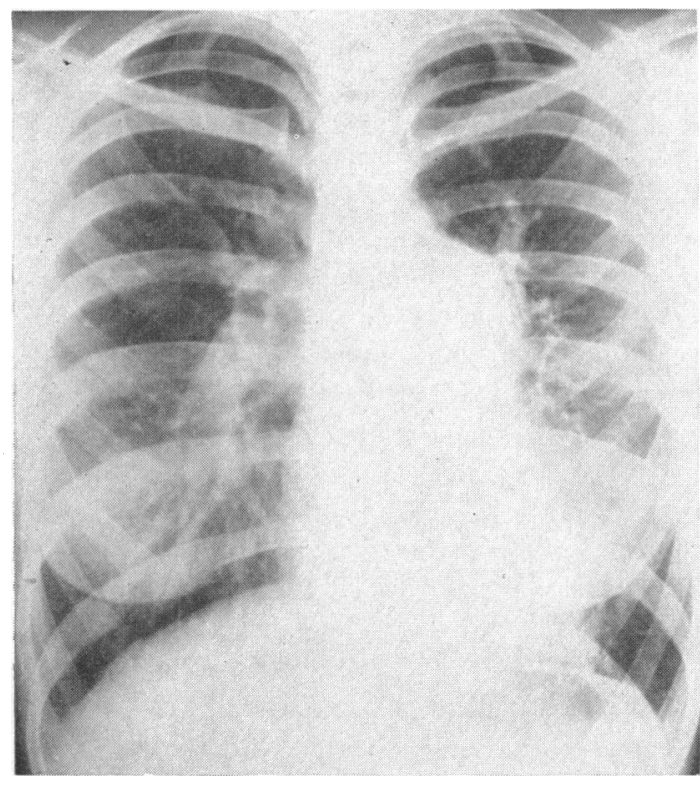

Fig. 9.-Eisenmenger's syndrome, showing very large main pulmonary artery, arterial root shadows, and clear lung peripheries; but absence of zonal differences in vasculature or of evidence of pulmonary interstitial œdema.

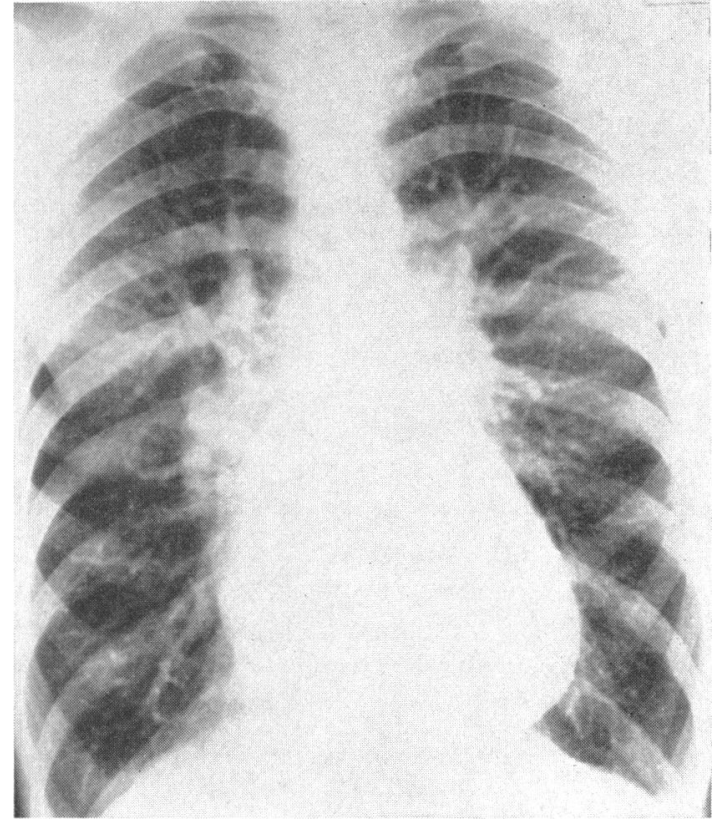

FIG. 10.-Atrial septal defect with low PVR and pulmonary systemic flow ratio of $4: 1$. Lung roots of arterial type but with somewhat ill-defined upper and outer borders because large hilar veins cross them, and the lobar and segmental arteries to upper lobes are markedly enlarged. Peripheral lung vessels are enlarged, but without zonal difference. No evidence of pulmonary interstitial œdema.

The main and lobar arteries increase in size, but the large veins decrease in size, and the segmental arteries and veins to the basal segments and to the middle lobe and lingula constrict. As a result of these changes the upper and outer boders of the lung roots become much better defined and arterial in character, while the lower ends of the root shadows become narrowed, conical, and curtailed, taking the appearance of blunt inverted cones, with a tail formed by the constricted descending arteries. The latter appearance is much more obvious on the right side where the heart shadow does not obscure the details. The clear area between the right root shadow and the right border of the heart may again become evident (Fig. 7 and 8). The region lateral to the right atrium becomes clearer because of the constriction of the segmental and subsegmental arteries and veins to the basal segments, the middle lobe, and the lingula.

The writer has indicated these two types of root shadow as venous and arterial because of their strikingly different appearances and their diagnostic importance. The arterial type of root shadow is often associated with veno-arterial constriction in the lower zones, a large main pulmonary artery and a high pulmonary vascular resistance and pulmonary arterial pressure, whereas these features are usually absent when the root shadow is of venous type. The main pulmonary artery may, however, be surprisingly large even when the pulmonary vascular resistance and pulmonary arterial pressure are relatively low, and when the root shadow is of venous type (Fig. 1a and 5a). In both situations the features of pulmonary interstitial œdema may be present or absent, though loss of translucency in the lower zones tends to be less in mixed than in venous hypertension (Fig. 1a and 7). This does not appear to be true of septal lines or of the lobular pattern.

In pure pulmonary arterial hypertension the main pulmonary artery is prominent and the root shadows are large, well-defined, and of arterial configuration. Their lower ends are often con- 
stricted and shortened, while a clear space is frequently visible between the right root shadow and the right atrium (Fig. 9).

Differential Radiological Diagnosis of the Varieties of Pulmonary Hypertension and of Pulmonary Pleonamia

Pulmonary Venous Hypertension. There is enlargement of the left atrium. Enlargement of the right ventricle tends to be slight and of the pulmonary artery slight or moderate, though it may be considerable (Fig. 1a and 5). The aorta is normal or slightly reduced in size. The root shadows are of venous type. Loss of translucency and opacification of the interlobular septa may or may not be present, depending upon the pulmonary venous pressure at the time of radiography. Dilatation of the medium-sized and small arteries and veins of the upper zones is frequently present, but the corresponding vessels of the lower zones are normal in calibre and are not constricted. The fact that the vessels in the upper zone are larger than those in the lower zone is of diagnostic value, for normally this situation is reversed. In mild cases the radiograms may be almost normal, whereas in severe ones all the features of pulmonary venous hypertension are florid (Fig. 1). Remarkable resolution of the root shadow changes, and of those due to pulmonary interstitial œdema, may follow rest (Fig. 5), or valvotomy.

Pulmonary Mixed Hypertension. There is enlargement of the left atrium. Enlargement of the right ventricle and main pulmonary artery, especially the latter, is usually greater than in pulmonary venous hypertension. The root shadows are of more arterial type, depending upon the severity of the rise in the pulmonary vascular resistance (Fig. 7 and 8). When the latter is high, well-marked arterial root shadows are obvious. Lower zonal arteriovenous constriction is often present and contrasts with the dilatation in the upper zone. Loss of translucency and opacification of the interlobular septa may or may not be present. Septal lines are often well seen, but loss of translucency in the lower zones tends to be less marked than in pulmonary venous hypertension. The most important features that distinguish mixed from venous pulmonary hypertension are the arterial type of root shadow and the lower zonal constriction of the medium-sized and small vessels.

Pulmonary Arterial Hypertension. There is not likely to be enlargement of the left atrium. The radiological appearances vary with the cause of the hypertension.

In primary pulmonary hypertension there is variable enlargement of the right ventricle and usually considerable enlargement of the main pulmonary artery. The root shadows are large and clearly defined, with a curtailed lower end. The clear space between the right root shadow and the heart is usually evident. The root shadows are, therefore, of arterial type. The peripheral vessels are small in all zones of the lung field and the changes of pulmonary interstitial œdema are absent, so that the lung fields are clear. The aorta is small.

When pulmonary arterial hypertension is secondary to an abnormal communication between the atria, the ventricles, or the aorta and pulmonary artery, variable radiological appearances may result. When the resistance to flow through an interventricular or aortopulmonary communication is no greater than it is through the normal aortic pathway (non-restrictive communication, Harley, 1960) and when no pulmonary stenosis is present, the systolic pressures in the aorta and pulmonary artery must always be identical, because the defect itself causes no pressure gradient. In these circumstances the pulmonary and systemic flows depend entirely upon the relative peripheral resistances of the pulmonary and systemic circuits. Either may be greater, or the two may be equal. The lung fields may, therefore, show the features of increased, normal, or decreased flow although the pulmonary arterial and aortic systolic pressures are the same as each other in each case. When an atrial septal defect or anomalous pulmonary veins are present, or when a communication between the ventricles or great vessels is restrictive and, therefore, creates a pressure gradient, the pulmonary 
arterial pressure can only reach or exceed systemic levels if the pulmonary vascular resistance equals or surpasses the systemic resistance. In this event the pulmonary flow will be decreased.

From what has been said it is evident that very different radiological appearances are found with pulmonary arterial hypertension due to congenital communications and full details cannot be given here. A feature common to all varieties is enlargement of the right ventricle and of the main pulmonary artery and its right and left branches. The root shadows are of arterial type, but the appearances of the medium-sized and small vessels is variable; they may be inconspicuous, normal, or enlarged, depending upon the pulmonary flow. Loss of translucency and opacification of the interlobular septa will not be seen. The size of the aorta varies with the site and size of the abnormal communication, and with the systemic flow. In cases of atrial septal defect the aorta is small (Fig. 9), in non-restrictive interventricular septal defects it may be normal or small, in restrictive interventricular septal defects it is usually normal, and in a large persistent ductus it is often bigger than normal, while the ductus itself may be visible radiologically, giving a double outline to the aortic arch (Wood, 1958). When the Eisenmenger syndrome is occasioned by an atrial septal defect the right pulmonary artery is sometimes disproportionately large (Fig. 9).

Pulmonary Pleonamia. Large pulmonary flows due to left-to-right intracardiac or aortopulmonary shunts are associated with large main, right, and left pulmonary arteries, together with enlargement of the lobar, segmental, and peripheral branches (Fig. 10). The pulmonary veins also dilate to accommodate the increased flow. The root shadows are largely of arterial type, but if the flow is very great they may show loss of definition of their upper and outer margins, caused by the large size of the hilar veins and of the origins of the segmental arteries, and even the clear space between the heart and the root shadow of the right lung may be encroached upon (Fig. 10). The medium sized vessels in the lower zones are somewhat larger than those of the upper zones, the opposite of the situation in pulmonary venous hypertension and pulmonary mixed hypertension. Slight left atrial enlargement may be seen in cases of ventricular septal defect or persistent ductus, in which conditions the left ventricle may also be enlarged. When an atrial septal defect is present the right atrium and ventricle are large, but the aorta is small. The aorta is usually normal in size when there is an interventricular communication, and may be enlarged when there is an aorto-pulmonary one.

\section{SUMMARY}

The significance and character of the radiological changes caused by pulmonary venous hypertension are discussed, with special reference to the lobular pattern and the root shadows.

Venous, mixed, and arterial varieties of pulmonary hypertension are defined. Pulmonary venous hypertension is a state in which the pressure in the pulmonary venous system is high, while the pulmonary vascular resistance remains normal, or increases only slightly, and any rise in pulmonary arterial pressure is passive. The root shadows are of venous type and loss of translucency and opacification of the interlobular septa are likely to be present, especially when the patient is active. Dilatation of the vessels to the upper zone is common, but constriction of those to the lower zone is not seen.

Pulmonary mixed hypertension occurs when a raised pulmonary vascular resistance, of spastic or structural origin, complicates pulmonary venous hypertension to produce a disproportionate rise in the pulmonary arterial pressure. The root shadows are of arterial type. Lower zonal constriction is often present and contrasts with the dilatation of the vessels in the upper zone. Opacification of the interlobular septa is often seen, but loss of translucency in the lower zone tends to be less obvious than in pulmonary venous hypertension.

Pulmonary arterial hypertension occurs whenever a raised pulmonary arterial pressure occurs without obstruction to pulmonary venous drainage. The radiological changes vary with the under- 
lying cause, but the features of a raised pulmonary venous pressure are absent, constriction of peripheral vessels is not zonal, and the root shadows are of arterial type.

Opacification of the interlobular septa causes horizontal lines and a lobular pattern on the radiogram. This opacification may be due to œdema, when it is reversible, or to structural changes, when it is irreversible.

The appearances caused by pulmonary pleonæmia are briefly described and are contrasted with those produced by the three types of pulmonary hypertension.

My grateful thanks are due to Dr. W. W. Phillips, Dr. J. C. Meek, and Dr. G. de B. Hinde for their helpful advice, to Professor J. Gough for the reproduction of the thin section of lung, and for his helpful criticisms, to Drs. T. E. Parry and F. K. Storring for the photo-micrographs, and to Mr. R. Marshall and Mr. Leighton Williams for the reproductions.

\section{REFERENCES}

Balchum, O. J., Gensini, G., and Blount, S. G., Jr. (1957). J. Lab. clin. Med., 50, 186.

Carmichael, J. H. E., Julian, D. G., Jones, G. P., and Wren, E. M. (1954). Brit. J. Radiol., $27,393$.

Davies, L. G., Goodwin, J. F., Steiner, R. E., and van Leuven, B. D. (1953). Brit. Heart J., $15,393$.

Dock, W. (1947). New Engl. J. Med., 236, 773.

Doyle, A. E., Goodwin, J. F., Harrison, C. V., and Steiner, R. E. (1957). $\quad$ Brit. Heart J., $19,353$.

Fleischner, F. G., and Reiner, L. (1954). New Engl. J. Med., 250, 900.

Fleming, R., and Simon, M. (1958). J. Fac. Radiol. (Lond.), 9, 33.

Goodwin, J. F. (1958). Brit. J. Radiol., 31, 174.

- Hollman, A., and O'Donnell, T. V. (1958). Lancet, 2, 1251.

- Steiner, R. E., and Lowe, K. G. (1952). J. Fac. Radiol. (Lond.), 4, 21.

Gough, J. (1955). Lancet, 1, 161 .

(1957-58). The Harvey Lectures, Series, 53, 171. New York, Academic Press.

Grainger, R. G. (1958). Brit. J. Radiol., 31, 201.

- and Hearn, J. B. (1955). J. Fac. Radiol. (Lond.), 7, 66.

Harley, H. R. S. (1960). Modern Trends in Cardiac Surgery. London, Butterworth and Co.

Harrison, C. V. (1958). Brit. J. Radiol., 31, 217.

Kerley, P. (1933). Brit. med. J., 2, 594.

(1951). in Shanks, S. C., and Kerley, P. A Textbook of X-ray Diagnosis by British Authors. 2nd ed., Vol. 2, p. 404. London, Lewis.

Miller, W. S. (1947). The Lung. 2nd ed. Springfield, Ill., Thomas.

Parker, F., Jr., and Weiss, S. (1936). Amer. J. Path., 12, 573.

Parry, T. E., and Storring, F. K. (Personal communication, to be published.)

Rossall, R. E., and Gunning, A. J. (1956). Lancet, 1, 604.

Short, D. S. (1955). Brit. Heart J., 17, 33.

(1956). Brit. Heart J., 18, 233.

Simon, M. (1958). J. Fac. Radiol., Lond., 9, 25.

Steiner, R. E. (1958). Brit. J. Radiol., 31, 188.

, and Goodwin, J. F. (1954). J. Fac. Radiol., Lond., 5, 167.

Wade, E. G., MacKinnon, J., and Vickers, C. F. H. (1956). Brit. Heart J., 18, 458.

Whitaker, W., and Lodge, T. (1954). J. Fac. Radiol., Lond., 5, 182.

Wood, P. (1958). Brit. med. J., 2, 701, 755. 